\title{
New Therapeutic Approach to Primary Stabbing Headache
}

\author{
Gaelle K. Ngonga, Daniela Carli, Paola Zavarise, Stefano Vollaro, Giorgio Dalla Volta \\ Clinical Institute Città di Brescia, Brescia, Italy \\ Email: ngonga.gaelle@gmail.com
}

Received 4 April 2016; accepted 18 April 2016; published 22 April 2016

Copyright (C) 2016 by authors and OALib.

This work is licensed under the Creative Commons Attribution International License (CC BY). http://creativecommons.org/licenses/by/4.0/

(C) (i) Open Access

\begin{abstract}
Primary stabbing headache has been known for several decades. However, the pathophysiology of this disease is not still understood. It is clinically characterized by single episodes or short-lasting series of stabbing pain recurring from once to many times per day in an irregular pattern. The diagnosis is clinical and any other possible cause should be excluded. The therapeutic approach indicates Indomethacin as the first choice of treatment, although case reports describe a therapeutic response to Melatonin, Celecoxib, Nidedipine, Paracetamol and Gabapentin. We describe the case of 5 patients with diagnosis of primary stabbing headache without comorbidity of anxiety or depression that shows a partial response to Indomethacin, but do respond to Benzodiazepines. There is little on the use of Benzodiazepines in primary headaches. They are used in patients with chronic migraine when there is a comorbidity of anxiety and depression. The unexpected efficacy of these molecules in our patients is encouraging and represents a hint to think about using these molecules in other types of primary headaches.
\end{abstract}

\section{Keywords}

Primary Stabbing Headache, Indomethacin, Benzodiazepines

Subject Areas: Neurology

\section{Introduction}

Primary stabbing headache (PSH) was first described in 1964 by Lasche as "ophthalmodynia periodica" [1]. Many other terms were successively coined to describe this syndrome, including "jabs and jolts syndrome" by Sjaastad et al. [2] and "ice pick-like headache" by Raskin and Schwartz [3]. The international classification of headache disorders, 3rd edition (beta version) had modified the terminology used for this entity from "idiopathic stabbing headaches" to "primary stabbing headaches" classified under chapter 4 "other primary headaches", and removed the criteria requiring stabs to be limited to the V1 distribution area [4]. 
The head pain is characterized by episodes of single or series of stabbing pain that last few seconds and occur from one to many times per day without a regular temporal pattern. The episodes involve the trigeminal or more frequently the extratrigeminal region. Moreover, the pain may shift from an area to another, in either the same or the opposite hemicranium. In addition, the pain has a fixed location in only one third of the patients in general [4]. For the diagnosis of PSH, it is mandatory that any other possible underlying disorder is ruled out.

Indomethacin represents the principal option for treating PSH despite therapeutic failure in up to $35 \%$ of the cases. Recent reports showed that Cyclooxygenase-2 (COX-2) inhibitors, Gabapentin, Nifedipine, Melatonin and Paracetamol may also be effective [5].

We describe the case of 5 patients suffering of PSH following the criteria of the ICHDIII beta that respond only partially to Indomethacin but do respond to Benzodiazepines.

\section{Case Report}

In the following, we present the symptoms and treatment of five patients who suffered of PSH. Table 1 gives an overview of the patients data.

The first patient was a 47-year-old woman assessed in 2012. The patient had been suffering from stabbing right temporal headache that appeared several times a day since 2 months. The neurological examination consisting in assessing the higher nervous functions, cranial nerves, motor ans sensory system and reflexes, was normal. A brain MRI and Angio MR were entirely normal. A five days treatment with Indomethacin $50 \mathrm{mg}$ bid reduced partially the pain. She was therefore treated with bromazepam from 0.5 to $3 \mathrm{mg}$ tid. The headache resolved after a few days of therapy and did not recur even after the discontinuation of the therapy 4 months after.

The second patient was a woman aged 58 assessed in 2013. She presented since 20 days a series of stabbing headache bouts of which each lasted no more than a few seconds. Each of the bouts had an overall duration of at most 1 minute. The episodes had an average frequency of $3-4$ times per day without associated symptoms. The patient did not suffer from any other type of primary headache. Neurological examination and brain MRI and Angio MR were entirely normal. A thermography was performed to study the autonomic nervous system involvement, resulting in no sympathetic activation in both the first and the second trigeminal branch. The patient was administered Indomethacin $50 \mathrm{mg}$ twice per day for a duration of 5 days and had a partial improvement. Thereafter, Bromazepam was prescribed. The dosage of this medication was increased slowly from $0.5 \mathrm{mg}$ to 3 mg three times per day. The symptom recovery occurred within a few days.

The third patient was a 54-year-old man assessed in 2013 for periorbital stabbing pain recurring 3 to 5 times per day over the prior 18 months without autonomic associated symptoms. A brain neuroimaging performed prior to the examination showed no abnormalities. The neurological examination performed during the medical visit was normal. A treatment with Indomethacin $50 \mathrm{mg}$ bid for 5 days was introduced with no result. The patient was therefore administered Bromazepam slowly increased up to $3 \mathrm{mg}$ tid with an almost complete resolution of the stabbing pain after few days. A month after the therapy was discontinued with worsening of the stabbing pain. Thus, the Benzodiazepine was reintroduced with benefit for 6 months. After two years the patient has sporadic episodes of short lasting stabbing pain. He is still on Bromazepam at the lower dosage of $1.5 \mathrm{mg}$ tid.

Table 1. Patients data.

\begin{tabular}{|c|c|c|c|c|c|}
\hline & Patient 1 & Patient 2 & Patient 3 & Patient 4 & Patient 5 \\
\hline Age & 47 & 58 & 54 & 58 & 33 \\
\hline Gender & Female & Female & Male & Male & Male \\
\hline Onset & 2 Months & 3 Weeks & 18 Months & 12 Months & A Month \\
\hline Number of crisis/day & $6-7$ & 10 & $3-5$ & 100 & 50 \\
\hline Frequency & Daily & Daily & Daily & Daily & Daily \\
\hline Response to indomethacin & Partial $^{*}$ & Partial $^{*}$ & None & Partial $^{*}$ & Partial $^{*}$ \\
\hline Associated headache & None & None & None & None & Migraine \\
\hline HAM-D & 4 & 5 & 3 & 4 & 4 \\
\hline Therapy & Bromazepam & Bromazepam & Bromazepam & Bromazepam & Alprazolam \\
\hline
\end{tabular}

*Partial: reduction of the frequency and or intensity of stabs less than 50\%; HAM-D: Hamilton Depression Rating Scale. 
The fourth patient was a man aged 58 and examined in 2014 for daily occurrences of highly recurrent right parieto-temporal headache without associated neuro-vegetative symptoms. The episodes have been occurring since a year. The neurological examination as well as the brain MRI and MRA were normal. The symptoms slightly reduce after a five days treatment with Indomethacin $50 \mathrm{mg}$ bid. Therefore Bromazepam $3 \mathrm{mg}$ tid was initiated with progressive and complete resolution after a month. Bromazepam was reduced to $2 \mathrm{mg}$ tid because of drowsiness, which disappeared with the tapering of the medication.

The fifth patient was a 33-year-old male with no clinical history except for sporadic attacks of migraine. He was examined 10 months ago for a right temporal stabbing headache with a severe short lasting pain occurring several hours per day since a month. Neurological examination and a neuroimaging scan were normal. Therapy with Indomethacin $50 \mathrm{mg}$ bid for five days reduced only partially the symptoms. A second treatment with Alprazolam $0.50 \mathrm{mg}$ tid was introduced. The treatment led to a complete resolution of the headache after only few days. The patient is asymptomatic after the discontinuation of the treatment.

All our patients had a normal score at the Hamilton Depression Rating Scale.

All the drugs used by patients were prescribed by physicians and bought in a pharmacy choose by the patient.

\section{Discussion}

PSH is one of the Indomethacin responsive headaches, which represent the first line treatment for this pathology [6]. The drug is effective in almost $75 \%$ of patients receiving it, although the efficacy is complete in only $51 \%$. Then, more than $35 \%$ of patients receiving therapeutic dosages of Indomethacin experience adverse effects and approximately $20 \%$ have to discontinue the drug [7]. Indeed the gastrointestinal, renal side effects or contraindications to Indomethacin (e.g. anticoagulation therapy) do limit it use.

In the patients who do not tolerate indomethacin or are not relieved by the medication, the management of PSH become a challenge. However other drugs that may be helpful in the treatment of PSH has been pointed out by other observations.

Melatonin on the strength of its possible pain-relieving properties, was administered to three patients with PSH to assess its effectiveness, in a study by Rozen [8]. The three patients were given different dosages of Melatonin (respectively 3, 9 and $12 \mathrm{mg}$ ). All the patients became asymptomatic and remained so for 2- to 4-months of follow-up.

A therapeutic approach with Celecoxib, a COX-2 specific inhibitor, was described by Piovesan et al. [9] in three elderly patients presenting PSH within 20 days of stroke onset. They responded to Celecoxib (100 mg bid), with full recovery from PSH within 6 days of first administration of the drug. After 60 days of treatment the drug was discontinued with the reappearance of painful symptoms in two patients. Celecoxib was reintroduced with complete disappearance of the pain. During a follow up of 120 days no one showed any further recurrence of the pain. A second interruption of Celecoxib did not cause the headache to recur.

Gabapentin (400 mg bid) was described by Franca et al. [10], as alternative drug in four patients with PSH not responding to 10 days treatment with indomethacin $50 \mathrm{mg}$ tid. The patients became asymptomatic after a few days ( 3 to 15 days) of therapy. The treatment was discontinued after 6 months without a return of the symptoms.

Slowrelease Nifedipine (90 $\mathrm{mg} /$ day) was effective in a case report described by Jacome [11]. The patient remained asymptomatic over a 3-year follow-up.

In a pediatric population, Fusco et al. [12] described a good response in 12 patients treated with paracetamol.

All our patients underwent a first therapy with Indomethacin 50 mg twice per day for 5 days with partial or no benefit. A Benzodiazepine treatment was subsequently introduced.

After only few days of treatment (3 to 10), they all had the complete recovery from their symptoms without collateral effects except a case of drowsiness that disappeared with lowering of the dosage of the molecule.

We chose to use a second line therapy with Benzodiazepine because of the gabaergic moduling of pain threshold of these molecules.

A reduction in the GABAA -mediated endogenous inhibitory control within the central nervous system leads to exaggerated pain and hyperalgesia [13]. The potentiation of GABAA receptor-mediated synaptic inhibition by Benzodiazepines reverses pathologically increased pain sensitivity in animal studies [14]. In mice and rats subtype-selective compounds targeting the alpha 2 and/or alpha 3 subunit of the GABAA receptor produce antihyperalgesia without sedation and without tolerance induction [15]. Thus the actions of Benzodiazepines are due to the potentiation of the neural inhibition that is mediated by GABA on type A GABA receptor system that 
is also the primary pharmacological target for many drugs.

\section{Conclusions}

There is not much in the literature on the treatment of PSH. The papers are all case reports which describe a therapeutic response to Indomethacin, Melatonin, COX-2 inhibitors, Nifedipine and Gabapentin. None of these therapies has been evaluated in controlled trials.

There is also little on the use of Benzodiazepines in primary headaches. They are commonly used with excellent results in patients with chronic migraine when there is a comorbidity of anxiety and depression. The mechanism for this improvement remains unclear. It may be due to the activity on anxiety and depression, or to the Gabaergic moduling of pain threshold of these molecules as explained before. This is the reason why we choose to use Benzodiazepines in a pathology that is scarcely affected by psychiatric conditions, and we select patients without anxiety or depression comorbidity, as shown by the normal score at the HAM-D.

Ours is a case report with few patients but the unexpected degree of efficacy of Benzodiazepines shown by our result is encouraging and represents a hint to think about using these molecules in other types of primary headaches.

A larger patients cohort is necessary to confirm our results.

\section{References}

[1] Lansche, R.K. (1964) Ophthmodynia Periodica. Headache, 4, 247-249. http://dx.doi.org/10.1111/j.1526-4610.1964.hed0403247.x

[2] Sjaastad, O., et al. (1979) Chronic Paroxysmal Hemicranial: Mechanical Precipitation of Attacks. Headache, 19, 3136. http://dx.doi.org/10.1111/j.1526-4610.1979.hed1901031.x

[3] Raskin, N.H. and Schwartz, R.K. (1980) Ice Pick-Like Pain. Neurology, 3, 203-205. http://dx.doi.org/10.1212/WNL.30.2.203

[4] Headache Classification Committee of the International Headache Society (IHS) (2013) The International Classification of Headache Disorders. Cephalalgia, 33, 629-808.

[5] Ferrante, E., Rossi, P., Tassorelli, C., Lisotto, C. and Nappi, G. (2010) Focus on Therapy of Primary Stabbing Headache. The Journal of Headache and Pain, 11, 157-160.

[6] Dodick, D.W. (2004) Indomethacin-Responsive Headache Syndromes. Current Pain and Headache Reports, 8, 19-26.

[7] Fuh, J.L., Kuo, K.H. and Wang, S. (2007) Primary Stabbing Headache in a Headache Clinic. Cephalalgia, 27, 10051009. http://dx.doi.org/10.1111/j.1468-2982.2007.01365.x

[8] Rozen, T.D. (2003) Melatonin as a Treatment for Idiopathic Stabbing Headache. Neurology, 61, 865-866. http://dx.doi.org/10.1212/01.WNL.0000082388.03466.E9

[9] Piovesan, E.J., Zukerman, E., Kowacs, P.A. and Werneck, L.C. (2002) COX-2 Inhibitor for the Treatment of Idiopathic Stabbing Headache Secondary to Cerebrovascular Diseases. Cephalalgia, 22, 197-200. http://dx.doi.org/10.1046/j.1468-2982.2002.00346.x

[10] Franca Jr., M.C., Costa, A.L. and Maciel Jr., J.A. (2004) Gabapentin Responsive Idiopathic Stabbing Headache. Cephalalgia, 24, 993-996. http://dx.doi.org/10.1111/j.1468-2982.2004.00791.x

[11] Jacome, D.E. (2001) Exploding Head Syndrome and Idiopathic Stabbing Headache Relieved by Nifedipine. Cephalalgia, 21, 617-618. http://dx.doi.org/10.1046/j.1468-2982.2001.00227.x

[12] Fusco, C., Pisani, F. and Faienza, C. (2003) Idiopathic Stabbing Headache: Clinical Characteristic of Children and Adolescents. Brain \& Development, 25, 237-240. http://dx.doi.org/10.1016/S0387-7604(02)00216-4

[13] Zeilhofer, H.U. (2005) The Glycinergic Control of Spinal Pain Processing. Cellular and Molecular Life Sciences, 62, 2027-2035. http://dx.doi.org/10.1007/s00018-005-5107-2

[14] Knabl, J., Zeilhofer, U.B., Crestani, F., Rudolph, U. and Zeilhofer, H.U. (2009) Genuine Antihyperalgesia by Systemic Diazepam Revealed by Experiments in GABAA Receptor Point-Mutated Mice. Pain, 141, 233-238. http://dx.doi.org/10.1016/j.pain.2008.10.015

[15] Knabl, J., Witschi, R., Hosl, K., Reinold, H., Zeilhofer, U.B., et al. (2008) Reversal of Pathological Pain through Specific Spinal GABAA Receptor Subtypes. Nature, 451, 330-334. http://dx.doi.org/10.1038/nature06493 